\title{
MOLECULAR DYNAMICS SIMULATIONS OF DISPLACEMENT CASCADES IN MOLYBDENUM
}

\author{
Richard Whiting Smith
}

\begin{abstract}
This report was prepared as an account of work sponsored by an agency of the United States Government. Neither the United States Government nor any agency thereof, nor any of their employees, nor any of their contractors, subcontractors or their employees, makes any warranty, express or implied, or assumes any legal liability or responsibility for the accuracy, completeness, or any third party's use or the results of such use of any information, apparatus, product, or process disclosed, or represents that its use would not infringe privately owned rights. Reference herein to any specific commercial product, process, or service by trade name, trademark, manufacturer, or otherwise, does not necessarily constitute or imply its endorsement, recommendation, or favoring by the United States Government or any agency thereof or its contractors or subcontractors. The views and opinions of authors expressed herein do not necessarily state or reflect those of the United States Government or any agency thereof.
\end{abstract}




\title{
Molecular Dynamics Simulations of Displacement Cascades in Molybdenum
}

\author{
Richard Whiting Smith
}

\begin{abstract}
Molecular dynamics calculations have been employed to simulate displacement cascades in neutron irradiated Mo. A total of 90 simulations were conducted for PKA energies between 1 and 40 keV and temperatures from 298 to $923 \mathrm{~K}$. The results suggest very little effect of temperature on final defect count and configuration, but do display a temperature effect on peak defect generation prior to cascade collapse. Cascade efficiency, relative to the NRT model, is computed to lie between 1/4 and $1 / 3$ in agreement with simulations performed on previous systems. There is a tendency for both interstitials and vacancies to cluster together following cascade collapse producing vacancy rich regions surrounded by interstitials. Although coming to rest in close proximity, the point defects comprising the clusters generally do not lie within the nearest neighbor positions of one another, except for the formation of dumbbell di-interstitials. Cascades produced at higher PKA energies (20 or 40 $\mathrm{keV}$ ) exhibit the formation of subcascades.
\end{abstract}




\section{Introduction}

Metals and metal alloys subjected to irradiation by fast neutrons exhibit microstructural evolution that drives deformation mechanisms such as irradiation creep and swelling, and produces embrittlement due to irradiation hardening. Microstructural evolution results from the formation of defect clusters and the flow of interstitials and vacancies to microstructural sinks. The rate at which this evolution proceeds is determined by the rate at which point defects are produced in displacement cascades resulting from the collision of fast neutrons with lattice atoms. In order to model microstructural evolution and its effects on material performance, it is first necessary to understand the dynamics of cascade formation and collapse. Key quantities that must be determined are the number of interstitials and vacancies produced in the cascades and the extent to which the defects cluster together during and immediately following the cascade event. Molecular dynamics (MD) simulations have been used to conduct detailed investigations of displacement cascades in a number of metallic and semiconductor systems [1-8]. The purpose of this paper is to report on molecular dynamics simulations of cascades in molybdenum.

\section{Computational Method}

Molecular dynamics simulations were conducted using a parallel version of the MDCASK program supplied by the Lawrence Livermore National Laboratory [1]. The program computes the time-dependent trajectories of the atoms in a region of crystal by solving Newtonian equations of motion for each atom, subject to the interatomic forces placed on them by their neighbors.

Displacement cascades are simulated by selecting an atom near the center of the computational cell to become a primary knock-on atom (PKA) and imparting to it some amount of kinetic energy at the start of the MD simulation. As the PKA collides with atoms in its vicinity, its original kinetic energy is distributed to its surroundings. Subsequent collisions between moving and stationary atoms result in the formation of the cascade. The calculation is continued until the defect population reaches a stable configuration. In the present investigation, cascades were tracked for a duration of roughly 10 picoseconds. PKA energies of 1, 2, 5, 10, 20 and $40 \mathrm{keV}$ were used and the PKA direction was taken as [135] in the BCC Mo lattice. Stoller has shown that for PKA energies above $1 \mathrm{keV}$, direction has little influence on cascade formation [9], so only a single direction was used in this work. The [135] direction was selected because it is unlikely that the PKA will channel. Prior to the introduction of the PKA, the computational cells were first equilibrated to an ambient temperature of either 298,573 or $923 \mathrm{~K}$. Equilibration was done using the velocity scaling algorithm in MDCASK. Three-dimensional periodic boundary conditions were applied to the computational cells and the cell dimensions were scaled to account for thermal expansion. Computational cell size was selected in accordance with previous work done on other systems [6,7] and to minimize any size effect that would result from 
temperature increases due to the dissipation of the PKA's kinetic energy. Cell size is given in Table 1. The temperature equilibration algorithm was switched off once the temperature had been equilibrated, prior to launching the PKA.

Table 1. Size of computational cells used in simulations.

\begin{tabular}{|c|c|c|}
\hline $\begin{array}{c}\text { PKA } \\
\text { Energy } \\
\text { (keV) }\end{array}$ & $\begin{array}{c}\text { No. BCC } \\
\text { unit cells. }\end{array}$ & $\begin{array}{c}\text { Total No. } \\
\text { atoms }\end{array}$ \\
\hline 1 & $28 \times 28 \times 28$ & 43,904 \\
\hline 2 & $35 \times 35 \times 35$ & 85,750 \\
\hline 5 & $47 \times 47 \times 47$ & 207,646 \\
\hline 10 & $59 \times 59 \times 59$ & 410,758 \\
\hline 20 & $74 \times 74 \times 74$ & 810,448 \\
\hline 40 & $93 \times 93 \times 93$ & $1,608,714$ \\
\hline
\end{tabular}

The interatomic potential used to describe the Mo system was constructed by joining together the Universal potential [11] at short range with a Finnis-Sinclair potential previously fit to equilibrium properties of Mo [12]. The connection was made with an exponential blend designed to maintain the continuity of energy and first derivative at the junction to both the Universal and Finnis-Sinclair regimes [13].

\section{Results and Discussion}

In order to improve statistics, five MD simulations were run at each PKA energy and cell temperature. Through out the cascade simulations, the defect counting algorithm of MDCASK was used to determine the number of vacancies and interstitials as well as their positions as a function of time. The average number of interstitial-vacancy pairs is plotted as a function of time and PKA energy at each starting temperature in figure 1. The curves display the characteristic rapid increase in defect number immediately following the PKA collision as adjacent atoms are knocked off of their lattice sites, and then a decay in defect number as vacancies and interstitials begin to recombine during the collapse of the cascade. As the event is concluded, the defect number approaches a fairly constant value that we take as the net yield of the cascade. It is important to point out that since periodic boundary conditions are used and there are no additional sinks in the simulation, if the calculations were to be extended indefinitely the total defect count would have to reduce to zero. If sufficient time were given for long range diffusion, the ultimate fate of each defect would eventually be to recombine with a partner of opposite type. There is simply no place else for them to go. In the actual material, 
recombination competes with absorption at sinks such as dislocations and voids. In this work, an arbitrary cutoff time between 6 and 8 ps is used to define the stable number of defects produced by the cascade.

Table 2 presents the maximum number of defects, time to maximum defect count and the stable number of defects for each simulation set. Also listed is the ratio of stable number relative to the maximum. Clearly at each temperature, the peak and stable number of defects increase with increasing PKA energy. There does not appear to be a significant temperature effect on stable defect number at constant energy, but the peak defect count does increase with increasing temperature. This increase probably reflects the fact that increased thermal vibration impedes the ability of the lattice to channel, reducing the number of sub-threshold collisions that do not produce defects. This observed temperature dependence should not suggest that the displacement threshold itself is temperature dependent. Zepeda-Ruiz, et al. have conducted detailed MD investigations into the threshold energy of vanadium, and found no temperature dependence [14]. They point out that no dependence should be expected since the displacement threshold is quite a bit larger than thermal energies even near the melting point.

Table 2 also presents the number of defects predicted by the NRT model [15] and the cascade efficiency, given by the ratio of stable number to NRT prediction. The NRT model is a modification to the Kinchin-Pease [16] approximation to the number of defects produced per collision, and gives the defect yield as,

$$
v(T)=\frac{0.8 T}{2 E_{d}}
$$

where $T$ is the PKA energy and $E_{d}$ the displacement threshold. Greenwood's Specter program [17] lists the displacement energy of Mo as $60 \mathrm{eV}$. The cascade efficiency values in Table 2 show that efficiency decreases slightly with increasing PKA energy and appears to be fairly insensitive to temperature. A value between $1 / 4$ and $1 / 3$ seems to be a reasonable approximation to use for the production efficiency needed by rate theory calculations of point defect balance and microstructural evolution. These observations are consistent with simulations performed on other pure metals $[4,6,10]$, and suggest that cascade efficiency itself is probably not strongly system dependent in monatomic metals. This insensitivity is not unexpected since cascade evolution is largely governed by close range interactions that can be treated as binary collisions. The details of the lattice structure and atomic bonding may not come into play until after the cascade has collapsed. It should be pointed out that the insensitivity should not be taken to imply that cascade efficiency would be unaffected by alloying. Alloying introduces differences in both the mass and size of the atoms, and MD simulations of low energy cascades in a Cu-Al binary alloy have demonstrated a change in defect production via 
replacement collisions to be caused by the mass and size difference of substitutional solute atoms [18]. For metallic systems in which constituent masses are similar, the effect of mass on collision dynamics is probably of secondary importance. The maximum energy transfer possible in a binary collision between particles of mass $M_{1}$ and $M_{2}$ is $\Lambda=4 M_{1} M_{2} /\left(M_{1}+M_{2}\right)^{2}$. When $M_{1}=M_{2}, \Lambda=1$, but even if $M_{1}$ is scaled to twice $\mathrm{M}_{2}, \Lambda$ drops only to 0.88 . This will have some impact on collision dynamics, but it is not expected to be overwhelming. The size difference could be more significant in that it disrupts the local symmetry of the lattice and can therefore affect mechanisms such as focussing, channeling and replacement collisions. It is anticipated that alloy effects for higher energy collisions on the development and collapse of the cascades will be smaller than the effects on subsequent evolutionary processes arising from atomic diffusion.

Table 2. Defect count for each simulation set.

\begin{tabular}{|c|c|c|c|c|c|c|}
\hline $\mathrm{T} / \mathrm{E}_{\mathrm{pka}}$ & Peak Count & $\begin{array}{c}\text { Peak Time } \\
\text { (ps) }\end{array}$ & Stable No. & Stable/Peak & NRT & Stable/NRT \\
\hline \multicolumn{7}{|l|}{$298 K$} \\
\hline $1 \mathrm{keV}$ & 26.8 & 0.33 & 2.6 & 0.10 & 6.7 & 0.39 \\
\hline 2 & 47.9 & 0.33 & 6.2 & 0.13 & 13.3 & 0.47 \\
\hline 5 & 111.3 & 0.39 & 13.2 & 0.12 & 33.3 & 0.40 \\
\hline 10 & 240.3 & 0.44 & 17.4 & 0.07 & 66.7 & 0.26 \\
\hline 20 & 451.7 & 0.50 & 38.5 & 0.09 & 133.3 & 0.29 \\
\hline 40 & 1088.3 & 0.99 & 69.8 & 0.06 & 266.7 & 0.26 \\
\hline \multicolumn{7}{|l|}{$573 \mathrm{~K}$} \\
\hline $1 \mathrm{keV}$ & 27.2 & 0.33 & 2.4 & 0.09 & 6.7 & 0.36 \\
\hline 2 & 51.0 & 0.33 & 5.8 & 0.11 & 13.3 & 0.44 \\
\hline 5 & 135.8 & 0.39 & 11.4 & 0.08 & 33.3 & 0.34 \\
\hline 10 & 282.0 & 0.44 & 17.4 & 0.06 & 66.7 & 0.26 \\
\hline 20 & 482.1 & 0.50 & 36.8 & 0.08 & 133.3 & 0.28 \\
\hline 40 & 1811.2 & 1.21 & 63.2 & 0.04 & 266.7 & 0.24 \\
\hline \multicolumn{7}{|l|}{$923 \mathrm{~K}$} \\
\hline $1 \mathrm{keV}$ & 32.6 & 0.33 & 3.6 & 0.11 & 6.7 & 0.54 \\
\hline 2 & 55.1 & 0.39 & 6.2 & 0.11 & 13.3 & 0.47 \\
\hline 5 & 136.7 & 0.39 & 8.6 & 0.06 & 33.3 & 0.26 \\
\hline 10 & 386.1 & 0.72 & 15.8 & 0.04 & 66.7 & 0.24 \\
\hline 20 & 683.0 & 0.83 & 34.2 & 0.05 & 133.3 & 0.26 \\
\hline 40 & 1842.5 & 1.10 & 68.5 & 0.04 & 266.7 & 0.26 \\
\hline
\end{tabular}


In addition to the point defect count and production efficiency, it is also important to understand the geometric configuration of the defects in space. Defect clustering not only affects their mobility but may also play a role in the nucleation of microstructural features such as voids and loops and in processes that contribute to irradiation hardening. Clustering behavior can be quantified by computing a distribution function expressing the number of defects that exist within clusters of varying size. Defects that lie within one nearest neighbor distance of one another are considered to belong to the same cluster. Cluster analysis can be most easily performed using a linked list, depth first search methodology described previously [19]. The analysis has been performed for both the stable interstitial and vacancy populations of each of the MD simulations. The results are tabulated in Tables 3 and 4.

Table 3. Average number of clusters of size 1-10

\begin{tabular}{|c|c|c|c|c|c|c|c|c|c|c|}
\hline \multicolumn{11}{|c|}{ Vacancies } \\
\hline$T / E$ & 1 & 2 & 3 & 4 & 5 & 6 & 7 & 8 & 9 & $10+$ \\
\hline \multicolumn{11}{|l|}{$298 K$} \\
\hline $1 \mathrm{keV}$ & 2.6 & & & & & & & & & \\
\hline 2 & 5.8 & 0.2 & & & & & & & & \\
\hline 5 & 12.2 & 0.6 & & & & & & & & \\
\hline 10 & 14.6 & 0.4 & 0.6 & & & & & & & \\
\hline 20 & 35.6 & 1.2 & & & & & & & & \\
\hline 40 & 65.8 & 1.4 & 0.2 & & & & & & & \\
\hline \multicolumn{11}{|l|}{$573 \mathrm{~K}$} \\
\hline $1 \mathrm{keV}$ & 2.6 & & & & & & & & & \\
\hline 2 & 5.4 & & & & & & & & & \\
\hline 5 & 10.6 & 0.4 & & & & & & & & \\
\hline 10 & 14.6 & 0.6 & & & & 0.2 & & & & \\
\hline 20 & 36.0 & 0.4 & 0.2 & & & & & & & \\
\hline 40 & 57.2 & 1.6 & 0.4 & 0.2 & & & & & & \\
\hline \multicolumn{11}{|l|}{$923 \mathrm{~K}$} \\
\hline $1 \mathrm{keV}$ & 3.8 & 0.2 & & & & & & & & \\
\hline 2 & 6.0 & & & & & & & & & \\
\hline 5 & 7.4 & 0.6 & & & & & & & & \\
\hline 10 & 15.0 & 0.4 & & & & & & & & \\
\hline 20 & 31.4 & 0.4 & & 0.2 & & & & & & \\
\hline 40 & 61.3 & 1.8 & 0.5 & & & & & & & \\
\hline
\end{tabular}


As shown in Table 3, there is only a very slight tendency for vacancies to cluster within the time frame of the initial cascade collapse. Most vacancies exist as isolated point defects, with only a small fraction combining into di-vacancies. Table 4 reveals that interstitials generally form in pairs. This does not result from the agglomeration of two atoms that have been displaced from their lattice sites during the collision, but merely reflects that fact that when a single self-interstitial comes to rest in the lattice it tends to adopt a dumbbell configuration with the nearest lattice atom. The atoms share the lattice site, each of them displaced slightly from it. Due to this method of accounting, interstitials outnumber vacancies two to one. There is some tendency for the interstitials to cluster into four- or sixmember structures. While not overwhelming, the clustering is clearly stronger in the interstitial population than it is for vacancies. This most likely results from the fact that interstitials are significantly more mobile than vacancies are, being able to take several diffusive steps during the time of the simulation. Clustering of both interstitials and vacancies appears to be less pronounced than in MD simulations of FCC materials such as Au [3]. A smaller tendency to cluster is consistent with previous simulations in other bcc metals $[6,10,20]$.

Although clustering at the end of the cascade, as defined by a strict nearest neighbor rule, is interesting, the ultimate goal of irradiation damage modeling is to determine whether or not the defects will annihilate or participate in microstructural evolution. This determination requires that much longer times be considered than those amenable to MD, and kinetic Monte Carlo (KMC) calculations are often performed to study the fate of the defects on a time scale consistent with atomic diffusion. While KMC simulations have not been done as part of this investigation, some degree of insight into whether the defects will annihilate or participate in the nucleation of larger structures can be gleaned from the images of the cascades themselves. Figure 2 illustrates the stable configuration of vacancies and interstitials for several cascades formed from a $20 \mathrm{keV}$ or $40 \mathrm{keV} \mathrm{PKA.} \mathrm{The} \mathrm{structures} \mathrm{tend} \mathrm{to} \mathrm{be}$ elongated, with vacancies concentrated near the center and interstitials surrounding the outside. The vacancies clearly group together, although the groups do not conform to the nearest-neighbor definition of a cluster used above. A quantitative measure of the degree to which interstitials and vacancies group together can be taken from the pair-correlation functions of the two species of point defects. To simplify the analysis, the correlation functions can be used to define an interaction parameter that reflects whether a point defect is surrounded more closely by like or unlike defects. Parameters for any given interstitial or vacancy are expressed as:

$$
P_{v}=\sum_{j=\text { OtherVacs }} \frac{1}{r_{i j}^{2}}-\sum_{k=\text { Ints }} \frac{1}{r_{i k}^{2}} \quad P_{i}=\sum_{j=\text { OtherInts }} \frac{1}{r_{i j}^{2}}-\sum_{k=\text { Vacs }} \frac{1}{r_{i k}^{2}}
$$


Table 4. Average number of clusters of size 1-10

\begin{tabular}{|c|c|c|c|c|c|c|c|c|c|c|}
\hline \multicolumn{11}{|c|}{ Interstitials } \\
\hline$T / E$ & 1 & 2 & 3 & 4 & 5 & 6 & 7 & 8 & 9 & $10+$ \\
\hline \multicolumn{11}{|l|}{$298 \mathrm{~K}$} \\
\hline $1 \mathrm{keV}$ & & 2.6 & & & & & & & & \\
\hline 2 & & 5.0 & & 0.6 & & & & & & \\
\hline 5 & & 8.6 & & 1.4 & & 0.4 & & 0.2 & & \\
\hline 10 & & 12.8 & & 1.6 & & 0.4 & & & & \\
\hline 20 & & 27.2 & & 2.6 & & 1.0 & & 0.8 & & 0.2 \\
\hline 40 & & 48.0 & & 7.0 & & 1.4 & & 0.4 & & 0.2 \\
\hline \multicolumn{11}{|l|}{$573 \mathrm{~K}$} \\
\hline $1 \mathrm{keV}$ & & 2.6 & & & & & & & & \\
\hline 2 & & 5.4 & & & & & & & & \\
\hline 5 & & 8.0 & & 0.8 & & & & 0.2 & & 0.2 \\
\hline 10 & & 10.4 & & 1.6 & & 0.8 & & & & 0.2 \\
\hline 20 & & 25.8 & & 3.0 & & 1.2 & & & & 0.4 \\
\hline 40 & & 40.6 & & 4.6 & & 1.4 & & 0.6 & & 0.8 \\
\hline \multicolumn{11}{|l|}{$923 \mathrm{~K}$} \\
\hline $1 \mathrm{keV}$ & & 3.6 & & & & 0.2 & & & & \\
\hline 2 & & 6.0 & & & & & & & & \\
\hline 5 & & 7.8 & & 0.4 & & & & & & \\
\hline 10 & & 10.2 & & 2.0 & & 0.4 & & & & \\
\hline 20 & & 23.2 & & 2.8 & & 1.0 & & & & 0.2 \\
\hline 40 & & 47.0 & & 4.3 & & 0.8 & & 0.8 & & 0.5 \\
\hline
\end{tabular}

A positive value of either quantity suggests the defect is more closely surrounded by members of the same species than its anti-defect. The average value of these quantities for the simulations conducted in this investigation is $\mathrm{P}_{\mathrm{i}}=0.1$ and $\mathrm{P}_{\mathrm{v}}=0.3$ suggesting that both interstitials and vacancies lie in closer proximity to members of their own species than their anti-defect. This condition supports both the formation of interstitial loops and void nuclei.

In addition to the production of the stable defect configuration, MD simulations are quite useful for understanding the dynamic processes that generate the cascade itself. Figure 3 presents six snapshots taken from a $20 \mathrm{keV}, 298 \mathrm{~K}$ cascade simulation at various points in time. The first defects to be produced lie in the upper cluster of figure $3 \mathrm{a}$. As time continues, the lower cluster develops as a 
sub-cascade, resulting from interactions with an energetic atom that escaped the initial damage region and traveled several lattice parameters, either by channeling or a replacement chain, before initiating a new collision. The cascade evolves to its maximum size in figure $3 \mathrm{~d}$ and then collapses toward its final configuration in figure $3 f$.

\section{Conclusions}

Molecular dynamics simulations of displacement damage cascades in bcc Mo reveal a number of interesting features:

Cascade damage increases with increasing energy, but cascade efficiency with respect to the NRT model decreases to a value between $1 / 4$ and $1 / 3$. This is consistent with MD simulations of previous metallic systems.

There appears to be little temperature dependence to the total number of stable defects produced in the cascade, but the peak defect count prior to cascade collapse does exhibit an increase with increasing temperature. It is proposed that this effect results from a reduction in channeling and focussing with increased thermal vibration.

With the exception of the di-interstitials, there is only a slight tendency for point defects to cluster into tight groups in which members are connected by one or two nearest neighbor distances. The defects do, however, agglomerate into vacancy or interstitial rich regions, where the point defects lie closer to each other than to their anti-defects, but not within one lattice constant. The vacancy rich regions tend to be surrounded by interstitials. The individual vacancy regions may mark the positions of sub-cascade formation.

Future efforts in this area are needed to determine the subsequent evolution of the defects on a longer time scale (especially the question of interstitial cluster mobility), and to understand whether or not cascade damage can be mitigated by alloying or microstructrural modifications. 


\section{Acknowledgements}

Many thanks are extended to Dr. L. A. Zepeda-Ruiz and Prof. B. D. Wirth for extensive assistance with MDCASK and the Mo interatomic potential.

\section{References}

1. T. Diaz de la Rubia, M. W. Guinan, J. Nucl. Mater. 174 (1990) 151.

2. $\quad$ A. F. Calder, D. J. Bacon, J. Nucl. Mater. 207 (1993) 25.

3. T. Diaz de la Rubia, N. Soneda, M. J. Caturla, E. A. Alonso, J. Nucl. Mater. 251 (1997) 13.

4. $\quad$ R. S. Averback, T. Diaz de la Rubia, Solid State Physics, 51 (1998) 281.

5. F. Gao, D. J. Bacon, P. E. J. Flewitt, T. A. Lewis, Mod. Sim. Mat. Sci. Eng. 6 (1998) 543.

6. E. Alonso, M. J. Caturla, T. Diaz de la Rubia, J. M. Perlado, J. Nucl. Mater. 276 (2000) 221.

7. R. E. Stoller, G. R. Odette, B. D. Wirth, J. Nucl. Mater. 251 (1997) 49.

8. R. E. Stoller, J. Nucl. Mater. 276 (1999) 22.

9. R. E. Stoller, Mat. Res. Soc. Symp. Proc. 650 (2001) R3.5.1.

10. W. J. Phythian, R. E. Stoller, A. J. E. Foreman, A. F. Calder, D. J. Bacon, J. Nucl. Mater. 223 (1995) 245.

11. J. F. Ziegler, J. P. Biersack, U, Littmark, The Stopping and Range of lons in Matter (Pergamon, New York, 1985).

12. G. Ackland, V. Vitek, Phys. Rev. B. 41 (1990) 10324.

13. B. D. Wirth, personal communication.

14. L. A. Zepeda-Ruiz, S. Han, D. J. Srolovitz, R. Car, B. D. Wirth, Phys. Rev. B 67 (2003) 13414.

15. M. J. Norgett, M. T. Robinson, I. M. Torrens, Nucl. Eng. Des. 33 (1975) 50.

16. D. R. Olander, Fundamental Aspects of Nuclear Reactor Fuel Elements, Technical Information Center Energy Research and Development Administration, Springfield, 1976.

17. L. R. Greenwood, R. K. Smither, "Specter: Neutron Damage Calculations for Materials Irradiations”, ANL/FPP/TM-197, Argonne National Laboratory, January 1985.

18. H. F. Deng, D. J. Bacon, Phys. Rev. B 48 (1993) 10022.

19. R. W. Smith, Z. Phys. D. 21 (1991) 57.

20. R. E. Stoller, J. Nucl. Mater. 233-237 (1996) 999. 

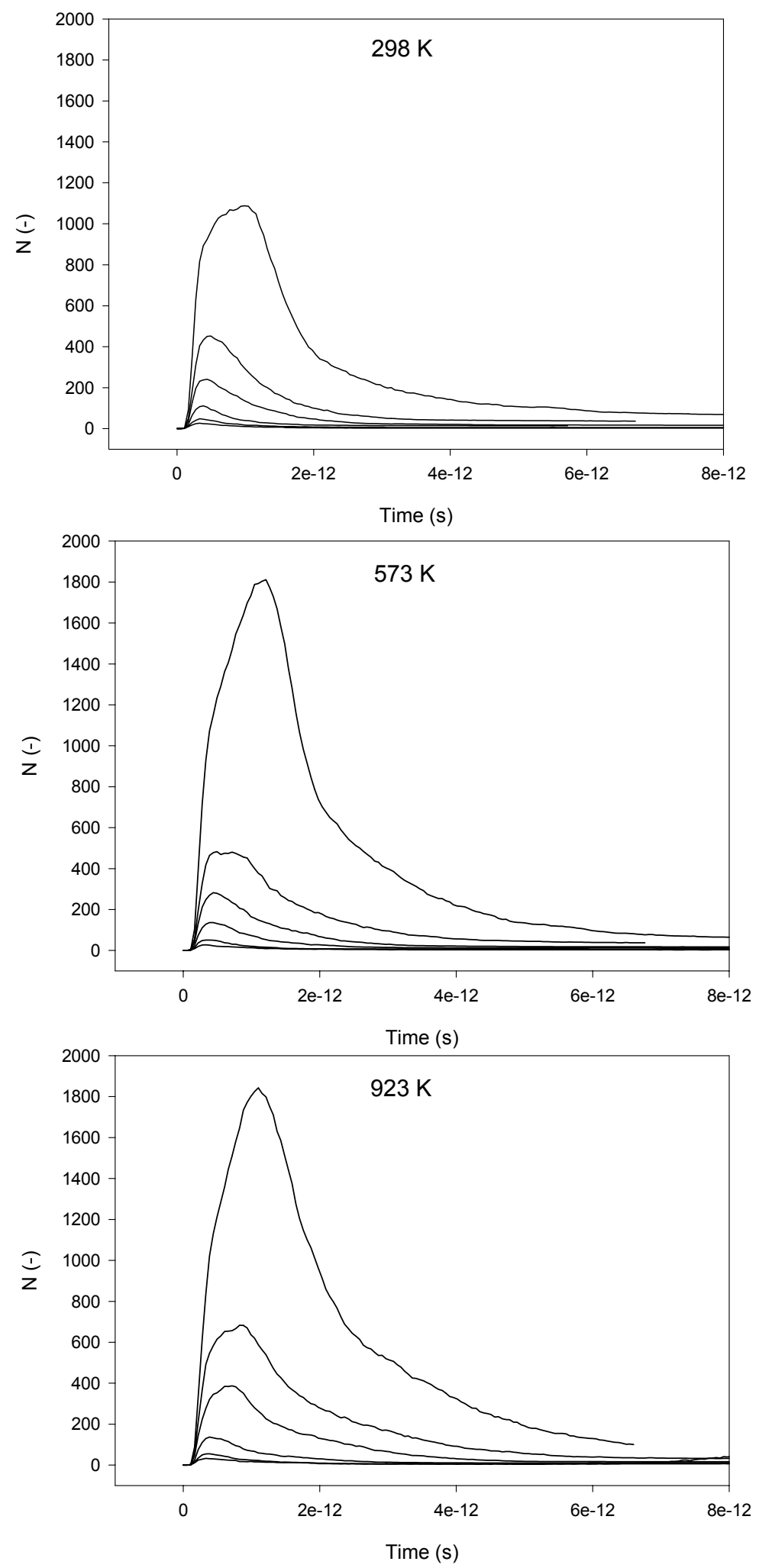

Figure 1. Time dependence of point defect number through out cascade event. Curves in each plot represent PKA energies of 1, 2, 5, 10, 20 and $40 \mathrm{keV}$. 


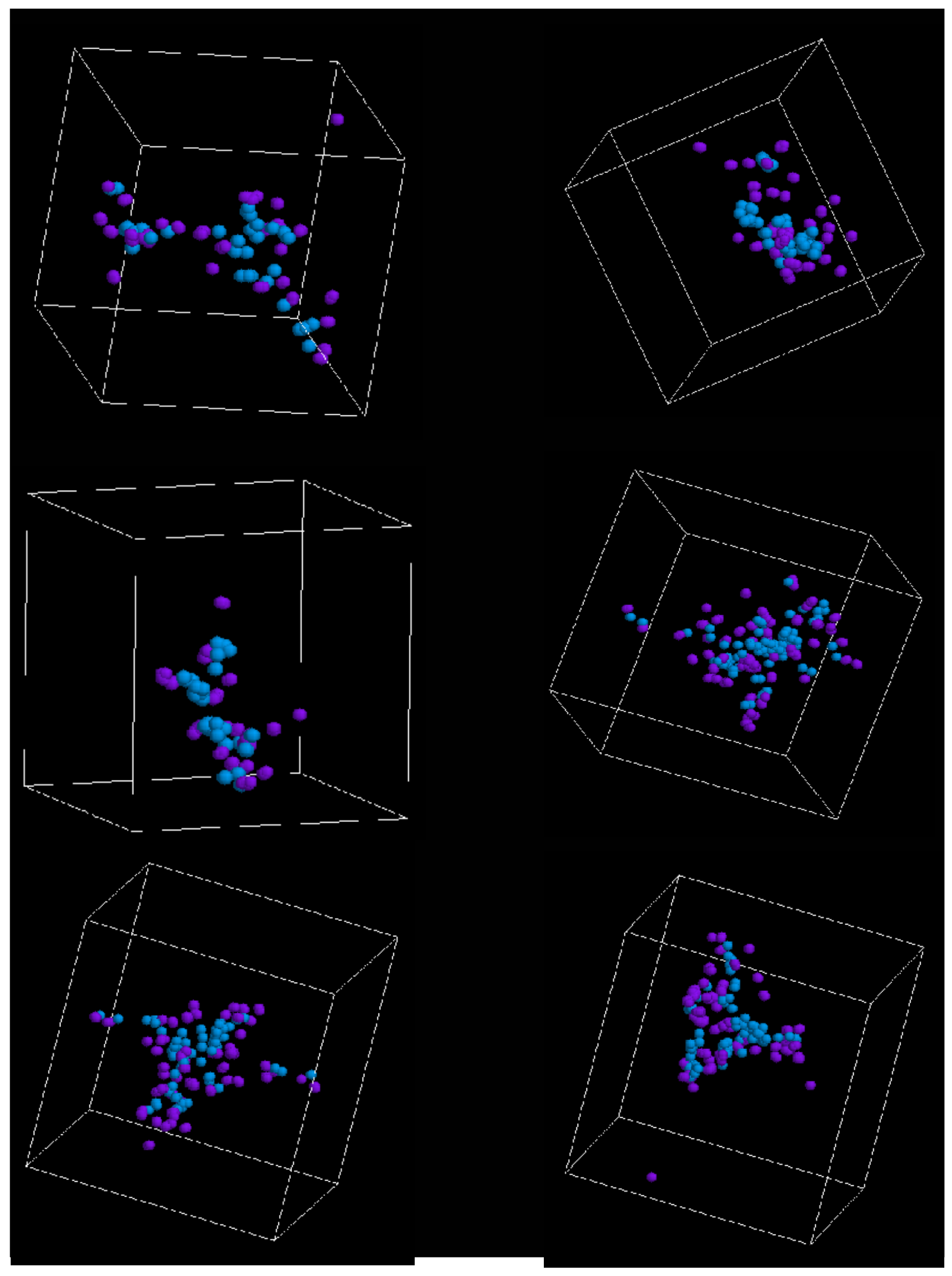

Figure 2. Typical stable point defect configurations following cascade events at 20 and $40 \mathrm{keV}$. Blue spheres represent vacancies. Purple represents interstitials. 


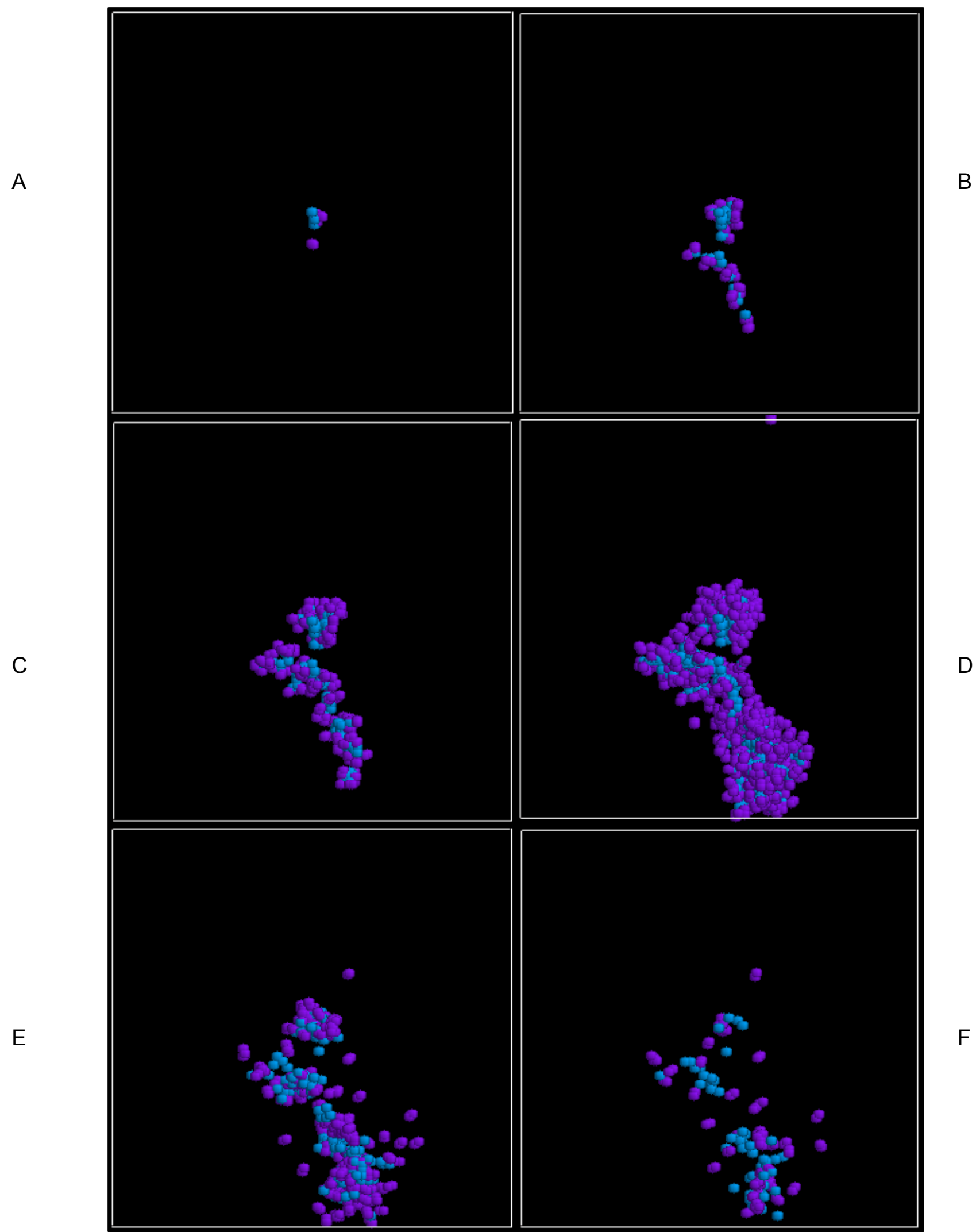

Figure 3. Time evolution of $40 \mathrm{keV}$ displacement cascade at $298 \mathrm{~K}$. Snapshots taken at times of (a) $0.08 \mathrm{ps}$, (b) $0.25 \mathrm{ps}$, (c) $0.4 \mathrm{ps}$, (d) $0.5 \mathrm{ps}$, (e) $1.1 \mathrm{ps}$, (f) $1.8 \mathrm{ps}$. 